\title{
Outcomes of Positive Airway Pressure for Sleep Apnea-Reply
}

\author{
Bruce Neal, $\mathrm{PhD}^{1}$; Zien Zhou, $\mathrm{MD}^{2}$; R. Doug McEvoy, $\mathrm{MD}^{3}$ \\ Author Affiliations \\ ${ }^{1}$ George Institute for Global Health, University of New South Wales Sydney, Sydney, \\ New South Wales, Australia \\ ${ }^{2}$ Ren Ji Hospital, Shanghai Jiao Tong University, Shanghai, People's Republic of \\ China \\ ${ }^{3}$ Adelaide Institute for Sleep Health, Flinders University, Adelaide, South Australia, \\ Australia
}

In Reply We agree with Drs Plante and Hoyt that using mean adherence to PAP among the relatively few patients remaining on treatment at 6-year follow-up in the trial by Peker et al overestimated mean adherence in that study. ${ }^{-}$In response to Plante and Hoyt, we obtained adherence data from the trial investigators (personal written communication, Yüksel Peker, $\mathrm{MD}, \mathrm{PhD}$, August 23, 2017), which estimates mean adherence to be 2.8 hours per day rather than 6.6 hours per day and recalculated the meta-regression. The corresponding revised metaregression for the outcome of major adverse cardiovascular events (MACE) is shown in the Figure and remains nonsignificant for the association between adherence to PAP and risk $(P=.17)$. The associations between adherence to PAP and risk for the MACE plus hospitalization for unstable angina outcome $(P=.56)$ and the cardiovascular death outcome $(P=.98)$ also remained nonsignificant. Revising the adherence subgroup analyses by placing the trial by Peker et al in the subgroup with adherence less than 4 hours per day did not substantively change the conclusions - there was still no definitive evidence of heterogeneity of effects of PAP on the MACE outcome in the trials with 4 or more hours per day adherence (relative risk, 0.43 [95\% CI, 0.22 to 0.85$]$ ) compared with the trials with less than 4 hours per day adherence (relative risk, 1.01 [95\% CI, 0.82 to 1.25$])(P$ value for homogeneity $=.06)$. The $P$ values for the comparisons between subgroups for other outcomes also remained nonsignificant (all $P>.26$ ).

Figure. 


\section{Figure. Updated Meta-Regression of Association of Mean Adherence to Positive Airway Pressure With Individual Trial Risk Ratios for Major Adverse Cardiovascular Events}

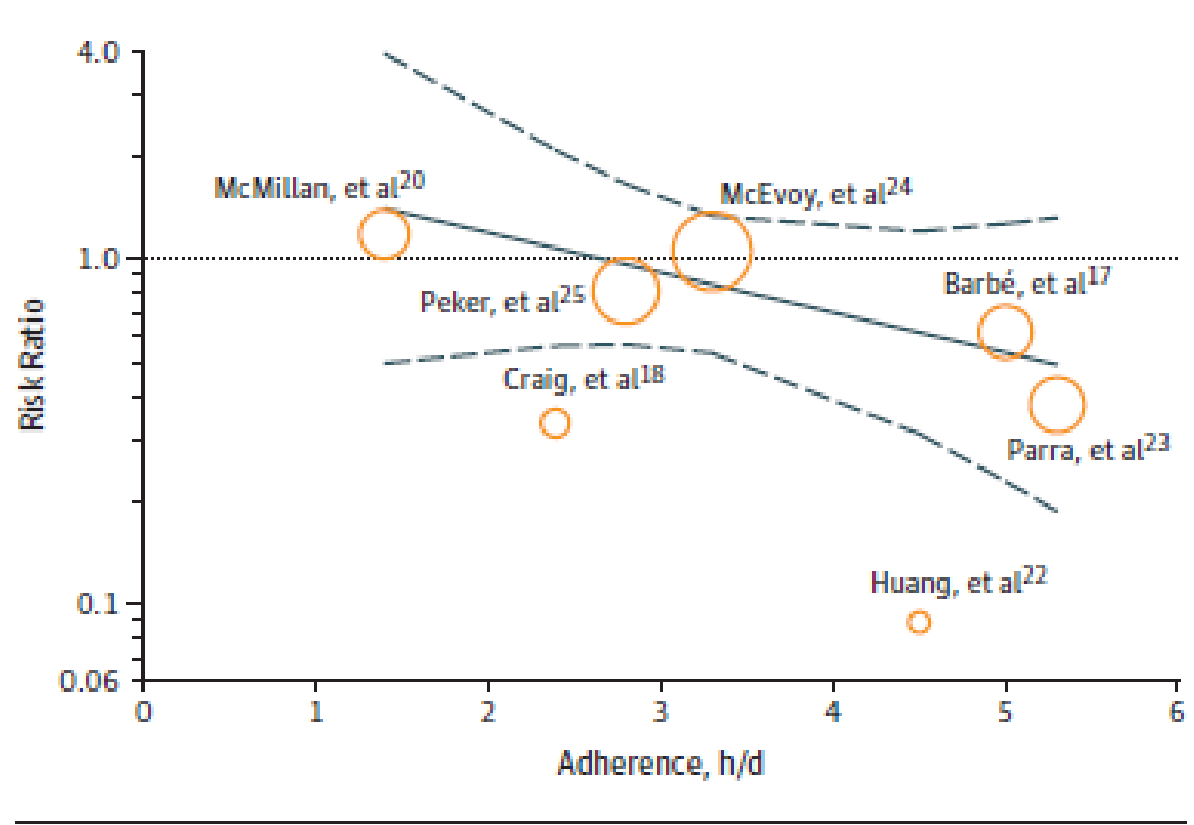

Circles represent trials with areas proportional to study size. Trials centered above the dotted line of unity are studies in which the relative risk of major adverse cardiovascular events with positive aiway pressure compared with control is greater than 1 , whereas trials centered below the dotted line have a relative risk of less than 1 . Refer to source article to access references cited in this figure. ${ }^{2}$

Updated Meta-Regression of Association of Mean Adherence to Positive Airway Pressure With Individual Trial Risk Ratios for Major Adverse Cardiovascular Events

Circles represent trials with areas proportional to study size. Trials centered above the dotted line of unity are studies in which the relative risk of major adverse cardiovascular events with positive airway pressure compared with control is greater than 1, whereas trials centered below the dotted line have a relative risk of less than 1 . Refer to source article to access references cited in this figure. ${ }^{2}$

In regard to the comments by Drs Javaheri and Campos-Rodriguez, we persist with our view that the evidence base is currently insufficient to support the use of PAP with the aim of reducing cardiovascular risk. ${ }^{3}$ We acknowledge that the available data are imperfect, but unless new studies show benefit, there is no reasonable basis for indicating to patients that treatment with PAP will prevent cardiovascular events. Observational analyses of the associations of sleep apnea with cardiovascular risks and cardiovascular outcomes are prone to confounding and an inadequate basis upon which to assure safety or efficacy of PAP for the prevention of cardiovascular events. Likewise, posthoc analyses of selected intermediate outcomes are a weak basis for defining benefit or harm for clinical outcomes. There are many examples of diseases for which observational findings have been shown to be inconsistent with trial results, even when clear benefits on intermediate risk markers have been demonstrated for interventions. In diabetes, for example, the withdrawal from the market of 
agents found initially to produce favorable effects on glycemia but ultimately to cause harmful effects on cardiovascular outcomes has resulted in an entirely new regulatory strategy for the approval of new therapies for diabetes. ${ }^{3}$ So, although the hypothesis laid out linking observational findings to intermediate outcomes and clinical events is interesting, it remains a hypothesis until sufficient evidence from adequately powered, well-conducted trials is generated to support it or refute it. Those data are currently unavailable and patient expectations should be set accordingly.

Section Editor: Jody W. Zylke, MD, Deputy Editor.

Article Information

Corresponding Author: Bruce Neal, PhD, George Institute for Global Health, Faculty of Medicine, University of New South Wales Sydney, Sydney, New South Wales, Australia 2050 (bneal@georgeinstitute.org.au).

Conflict of Interest Disclosures: The authors have completed and submitted the ICMJE Form for Disclosure of Potential Conflicts of Interest. Dr Neal reported receipt of a grant and personal fees from Janssen. Drs Neal and McEvoy reported receipt of support from the National Health and Medical Research Council (NHMRC) of Australia and being investigators on the SAVE trial, supported by grants from Philips Respironics and NHMRC with supplementary grants, equipment, or both from ResMed and Fisher\&Paykel. No other disclosures were reported.

References

1 .

Peker Y, Glantz H, Eulenburg C, Wegscheider K, Herlitz J, Thunström E. Effect of positive airway pressure on cardiovascular outcomes in coronary artery disease patients with nonsleepy obstructive sleep apnea: the RICCADSA randomized controlled trial. Am J Respir Crit Care Med. 2016;194(5):613-620.

2.

Yu J, Zhou Z, McEvoy RD, et al. Association of positive airway pressure with cardiovascular events and death in adults with sleep apnea: a systematic review and metaanalysis. JAMA. 2017;318(2):156-166.

3.

US Department of Health and Human Services Food and Drug Administration. Guidance for industry: diabetes mellitus - evaluating cardiovascular risk in new antidiabetic therapies to treat type 2 diabetes. https://www.fda.gov/downloads/Drugs/.../Guidances/ucm071627.pdf. Accessed September 22, 2017. 